(Aus der Universitätsklinik für Dermatologie und Syphilidologie in Wien [Vorstand: Hofrat Prof. Dr. G. Ri e hl].)

\title{
Über die Wasserbettmykose.
}

\author{
Von \\ Dr. Leo Kumer, \\ Assistent der Klinik. \\ (Eingegangen am 2. Aprit 1921.)
}

Krae peli n erwähnt 1901 als erster das Auftreten einer Epidemie von „Herpes tonsurans "im Gefolge der Dauerbadbehandlung, ohne auf die Eigenart dieser Erkrankung näher einzugehen. 6 Jahre später gibt uns Jacobi eine genaue klinische Schilderung dieser von ihm als "besondere Form der Trichophytie" aufgefaßten Dermatose.

Das Leiden beginnt nach Jacobis Besehreibung meist in der Genital- oder Axillargegend mit disseminierten, dunkelroten, wenig infiltrierten Papeln, die sich unter Größenwachstum oft über den ganzen Körper ausbreiten, wodurch Herde mit serpiginöser Begrenzung entstehen. An älteren kann man einen hellrot gefärbten Rand und ein dunkel lividrotes Zentrum unterscheiden, dazwischen befindet sich ein durch Epidermisauflagerungen weißlich getrübter Ring.

Jacobi beobachtete auch das Ergriffenwerden der Haut des Gesichtes, der Fandteller und Fußsohlen.

In den Auflagerungen ließen sich massenhaft Mycelfäden nachweisen.

$\mathrm{K}$ üster veröffentlichte die bakteriologischen Untersuchungsergebnisse über diese Erkrankung und, da er zu dem Schltusse kommt, daß der Erreger in die Gruppe der Askomyceten einzureihen sei, spricht er nicht mehr von einer Trichophytie, sondern von einem ,Ekzem ".

Beide Namen sind falsch, denn, da der Erreger nicht in die Trichophytongruppe gehört, kann man die Erkranking nicht als Trichophytie bezeichnen, andererseits ist es nach unseren heuizon Begriffen ebensowenig möglich, die in Frage stehende Dermatose als Ekzem aufzufassen, da eine ganz bestimmte Pilzart für die Entstehung der Erkrankung verantwortlich zu machen ist.

Außer den obengenannten Arbeiten ist keine weitere Veröffentlichung über die Erkrankung erfolgt und so ist es vielleicht nicht zu spät, einen unpassenden Namen in der dermatologischen Literatur für die Zukunft zu vermeiden.

Die von uns gebrauchte Bezeichnung, "Wasserbettmykose" dürfte wohl am zutreffendsten die wesentlichen Charakteristica der Erkrankung umfassen.

Wenn heute aus der Universitätsklinik für Dermatologie und Syphilidologie in Wien über diese Wasserbettmykose berichtet werden soll, so 
geschieht dies einerseits; weil die historische Stätte der Wirksamkeit Hebras - dem wir ja neben so vielem anderen auch die Wasserbettbehandlung verdanken - heute mit einem Stand von 29 Wasserbetten die größte Station dieser Art vorstellt und andererseits, weil die hierorts gemachten Beobachtungen in manchem von jenen Jacobis und $K \ddot{u}-$ sters abweichen, so daß auch die klinische Schilderung dieser Erkrankzung noch einer Ergänzung bedarf. Ja die Abweichung der in Freiburg und in Wien beobachteten Fälle ist so wesentlich, daß wir zwei verschiedene Formen der ihrer Ätiologie nach gleichen Erkrankung annehmen müssen.

Im folgenden soll die hierorts beobachtete Wasserbettmykose zusammenfassend beschrieben werden.

Klinik: Die Primärefflorescenz ist entweder ein Bläschen oder, viel seltener, eine stecknadelkopfgroße, lebhaft rote, kegelstumpfförmige Papel. Auch die Bläschen sind nicht über hanfkorngroß, liegen in den oberflächlichsten Schichten, prominieren wenig und kommen an Hautstellen mit dünner Hornschicht selten in ungeplatztem Zustande zur Beobachtung. Aber auch dann läßt sich aus dem zentral freien Epidermissaum oder aus der scharf ausgestanzten Vertiefung das ursprünglich vorgelegene Bläschen leicht erschließen. Die Farbe dieser Bläschen ist das Weil einer macerierten Haut, eine Weiterentwicklung zur Pustel ist wegen Kurzlebigkeit der Efflorescenz nur selten zu beobachten.

Nach Abstoßung der Blasendecke erscheint ein leicht geröteter Untergrund.

Bläschen und Papeln stehen selten allein, meist treten sie in Gruppen zusammen oder sind so dicht gestellt, daß die betreffende Hautpartie diffus erkrankt ist; niemals war jedoch ein Größenwachstum dieser Efflorescenzen zu beobachten.

Die Verschiedenartigkeit der Krankheitsbilder je nach der Lokalisation ist vor allem durch die wechselnde Mächtigkeit der Hornschicht an den erkrankten Stellen bedingt.

In der Kniegegend sind die Bläschen ab und zu wohl etwas größer, dafür desto kurzlebiger. Hier trifft man aber auch Knötchen, die lebhaft rot gefärbt sind, stark prominieren und eine flache Oberfläche haben, der weiße, macerierte, bröckelige Hornmassen aufliegen. Sind diese zur Abstoßung gelangt, so bleibt ein dünner Epidermissaum am Rande zurück. Anfänglich stehen Bläschen und Knötchen vereinzelt, dauert die Erkrankung an, so konfluieren sie vielfach zu Herden, in deren Umgebung immer wieder neue Efflorescenzen auftreten.

Gleiche Erscheinungsformen der Wasserbettmykose lassen sich ab und zu an anderen, im Wasser befindlichen Körperteilen feststellen, so an der Brust, am Rücken, Bauch, den Oberschenkeln und in der Achselhöhle. 
In den Interdigitalfalten der Hände kommen als Primärefflorescenzen nur Bläschen vor, die fast immer zu Gruppen zusammentreten; wir haben dann das Bild einer typischen interdigitalen Soormykose vor uns. Die Haut ist an der ergriffenen Stelle leicht gerötet, ihrer oberfläch. lichsten Epidermisschichten beraubt, ab und zu nässend und begrenzt von einem vielfach polycyclisch angeordneten, unterminierten Epidermissaum.

Auch an den Handtellern tritt die Mykose nur in Bläschenform auf und es gelingt hier leichter, solche noch unversehrt anzutreffen. Ist die Blasendecke abgehoben, so bleibt ein scharf ausgestanzter Substanzverlust der oberflächlichsten Epidermisschichten zurück, der durch die Maceration der überhängenden Blasendecken noch mehr in die Augen springt. Während an den Knien einzelstehende, schuppende Stellen oder aus der Konfluenz nur weniger Efflorescenzen entstandene Herde anzutreffen sind, ergreift die Erkrankung die Handteller oft diffus, in der macerierten Haut finden sich einerseits dicht nebeneinander gestellte Bläschen und Substanzverluste der Epidermis nach solchen, andererseits ist die Hornschicht in größerem Umfange zur Abstoßung gelangt und dann sieht man einen leicht geröteten, glatten Untergrund, der von weißlichen, macerierten Epidermismassen durchzogen und umsäumt ist. Von den Handtellern und Interdigitalfalten greift die Erkrankung auf die Beugeseite der Finger über, hier ist in gesunder Umgebung nur ein oder das andere Bläschen auffindbar, in anderen Fällen beobachtet man eine herpesähnliche Aussaat, hier und da ist die ganze Haut in diffuser Weise ergriffen. Ist die Erkrankung an den Handtellern sehr ausgebreitet, dann ist ab und zu ein Übergreifen von einzelnen Efflorescenzen auf den Handrücken zu beobachten.

Eine weitere Prädilektionsstelle für die Wasserbettmykose bildet die Umgebung der Fingernägel. Am Nagelwall sieht man hier und da dieselben Bläschen wie an den Handtellern. Von hier kriecht die Erkrankung in den Raum zwischen Nagelbett und freiem Nagelrand hinein und kann auch zu einer geringen lokalen Abhebung des Nagels von seiner Unterlage führen. Schwere Nagelveränderungen und Paronychien kamen nicht zur Beobachtung.

Damit ist auch die Lokalisation der Erkrankung, soweit wir sie beobachten konnten, beschrieben. Wenn man auch einzelne Bläschen an allen jenen Stellen der Haut, die mit warmem Wasser in Berührung sind, antreffen kann, wie am Stamm, in den Achselhöhlen, an den Oberschenkeln und in der Kniegegend, so sind gewisse Hautbezirke, wie die Interdigitalräume der Finger und die Vola manus besondere Lieblingsstellen der Pilzansiedlung.

Die Haare waren nie erkrankt und auch die Fußsohlen und die Interdigitalräume der Zehen nur in einem Falle von der Wasserbettmykose 
ergriffen. Da an Handtellern und Fußsohlen annähernd gleiche anatomische Verhältnisse herrschen, wäre auch ein öfteres Befallenwerden der letzteren zu erwarten. Daß dies in unseren Fällen nicht zutraf, mag vielleicht darin seine Erklärung finden, daß allen Patienten der Wasserbettstation zur Vermeidung der infolge Maceration der Fußsohlenepidermis auftretenden Schmerzen prophylaktisch die Fußsohlen öfters mit Teer eingepinselt werden.

Die Lokalisation der Wasserbettmykose läßt sich einerseits aus einer besonderen Disposition der Haut an diesen Stellen erklären und dies braucht uns ja, nachdem das Krankheitsbild der interdigitalen Soormykose bekannt geworden ist, nicht zu wundern; andererseits sind die Lokalisationsstellen gerade Hautpartien, die abwechselnd in der Luft und unter Wasser gehalten werden. Diese Abwechslung zwischen warmem Wasser und Luft scheint jene Bedingungen zu schaffen, die dem Erreger die Ansiedlung ermöglichen.

Die Beschwerden, welche die Erkrankung verursacht, sind minimale, auf Befragen geben die Patienten öfters ein leichtes Jucken an, doch meist sind sie sich ihrer abnormen Hautverhältnisse gar nicht bewußt.

Über Ausbreitung und Verlauf der Wasserbettmykose sollen die auszugsweise angeführten Krankengeschichten der an unserer Klinik beobachteten 11 Fälle Aufschluß geben:

1. 40 jährige Frau mit Lähmung beider unteren Extremitäten infolge Rückenmarktumors. Guter Ernährungszustand. Liegt seit 3 Tagen im Wasserbett.

Status pra esens: 6. I. 1921. Im linken dritten Interdigitalraum finden sich fünf knapp nebeneinanderstehende Bläschen, deren Blasendecke geplatzt ist und als macerierte weißliche Epidermis die erkrankte Stelle bedeckt. Pilzbefund negativ.

10. I. Im dritten linken Interdigitalraum ist durch Aufschießen neuer Bläschen der Herd zu Hellerstückgröße angewachsen. Die Blasendecke ist größtenteils zu Abstoßung gelangt, am polycyclisch begrenzten Rande jedoch als unterminierter Epidermissaum deutlich wahrnehmbar. Leichtes Juckgefühl.

16. I. Auch der zweite und vierte Interdigitalraum links sind an analogen Erscheinungen erkrankt. An der rechten Palma manus finden sich drei stecknadelkopfgroße, flach erhabene Bläschen mit macerierter, weißlicher Blasendecke.

20. I. Die Erkrankung greift von den Interdigitalräumen auf die benachbarte Partie der Vola manus über. In der Umgebung der am 15. I. aufgetretenen Bläschen an der Vola manus ist in herpesartiger Anordnung eine neue Aussaat erfolgt. Auch an den Beugeseiten der Finger zeigen sich einige Bläschen. Seit zwei Tagen sind auch an der rechten Vola manus einige Bläschen zu bemerken.

23. I. Am Nagelwall des ersten, dritten und vierten Fingers links sind einige Bläschen aufgetreten. Pilzbefund negativ.

30. I. Die alten Herde bestehen unvermindert fort. An beiden Handtellern ist auch die Gegend des Antithenars befallen worden. An den erkrankten Stellen des Nagelwalles ist die Hornschicht teilweise zur Abstoßung gelangt, die entsprechende Partie ist leicht gerötet und geschwollen. Die Erkrankung ist am linken ersten, zweiten und dritten Finger vom Nagelwall gegen die Fingerspitzen fort- 
gekrochen und hat die unter dem freien Nagelrande liegende Haut ergriffen. Die Nagelplatte ist etwas abgehoben und der frei werdende Raum von mazerierten Epidermismassen ausgefüllt. Pilzbefund positiv.

3. II. Außer den oben beschriebenen, weiter bestehenden Veränderungen zeigt die Haut der Pat. ein Ekzem, dessen Lokalisation jenen Körperstellen entspricht, die an der Wasser-Luftgrenze gehalten werden, und zwar: ein handtellerhreiter Streifen über der rechten Scapula, von dort sich bogenförmig über den Rippenbogen gegen die Nabelgegend hinziehend. Dieses Ekzem besteht aus stecknadelkopfgroßen, dicht nebeneinander gestellten, lebhaft roten, an den seitlichen Thoraxpartien auch follikulär gestellten Knötchen mit entzündlichem Hof. Auch durch Schaben mit einem Instrument kann man von diesen Knötchen nur spärliche Schuppenteile zu Untersuchungszwecken gewinnen. Der Pilabefund ist hier andanernd negativ.

14. II. In der rechten Achselhöhle sind einige, auf leicht entzündlicher Basis stehende Bläschen aufgeschossen, die Blasendecke ist größtenteils abgestoßen. Pilzbefund positiv.

20. II. Nach Trockenlegung der Pat. rasche Rückbildung der Krankheitserscheinungen.

2. 18jähriges Mädchen mit pyämischen Abscessen. Mittlerer Ernährungszustand. Liegt über ein Jahr im Wasserbett ohne bisher von der Mykose ergriffen worden zu sein.

Status praesens: 10. XII. 1920. In der Patellargegend links sind einige, bis linsengroße, flacherhabene Efflorescenzen aufgeschossen, deren Oberfläche mit macerierten, weißlichen, bröckeligen Schuppen bedeckt ist, die sich unschwer entfernen lassen.

14. XII. Aufschießen von Bläschen zwischen den alten Efflorescenzen, dadureh stellenweise Konfluenz.

20. XII. Nach Abstoßung der Schuppen ist eine im Niveau der Haut gelegene unregelmäßig begrenzte Stelle von lividroter Farbe und glatter Oberfläche zurückgeblieben. Der andauernd positiv gewesene Pilzbefund ist heate negativ. An der linken Hohlhand sind einige oberflächliche Bläschen aufgeschossen mit positivem Pilzbefund.

27. XII. Der Herd in der linken Patellargegend gelangt langsam zur Abheilung.

3. I. 1921. Die Erkrankung der linken. Hohlhand ist weiter fortgeschritten und hat den zweiten, dritten und vierten Interdigitalraum diffus ergriffen.

15. I. Die ganze Erkrankung ist trotz fortdauernder Wasserbettbehandlung unter Abstoßung der Hornschicht zur Abheilung gelangt. Pilzbefund sohon längere Zeit negativ.

3. 46 jährige Frau mit offener Fraktur des rechten Unterschenkels. Guter Ernährungszustand. Seit 5 Tagen im Wasserbett.

Status praesens: 4. I. 1921. An beiden Handtellern einige Bläschen mit positivem Pilzbefund.

25. I. Die Pat. hat ohne Wissen der Ärzte ihre Handteller mit Vaselin eingeschmiert. In der Umgebung der erst aufgeschossenen Bläschen sind nach und nach neue aufgetreten, derzeit sind beide Handteller diffus erkrankt und auch die Beuge- und Seitenflächen der Finger und die Interdigitalräume sind bis zu den Fingerspitzen diffus von der Mykose ergriffen. Man sieht Bläschen neben Bläschen. Die Blasendecke ist allerdings in den meisten Fällen bereits geplatzt. Daneben sind größere Epidermispartien zur Abstoßung gelangt, es zeigt sich dann ein leicht geröteter Grund, dem unregelmäßig begrenzte, mazerierte, weißliche Epidermisbrocken und Leisten aufliegen. An den polycyclisch begrenzten Randpartien ist ein unterminierter Epidermissaum gelegen. 
20. II. Die Erkrankung greift in einzelnen Ausläufern auch auf die Handrücken über. Außerdem bemerkt man seit einigen Tagen an der Haut der Brust und an beiden Oberarmen zahlreiche, stecknadelkopfgroße, lebhaft rote, stark prominente, kegelstumpfförmige Knötchen, an deren Oberfläche die Hornschicht zur AbstoBung gelangt ist. Während. an den unteren Brustpartien die Mehrzahl der Knötchen einzeln steht, treten sie besonders in der vorderen Achselfalte und in den angrenzenden Partien der Oberarme und Brust zu streifenartigen Herden zusammen. Der Aufbau dieser Herde entsteht immer nur durch Apposition einzelner Knötchen, zwischen denen dann eine seichte Furche liegt, niemals aber durch peripheres Weiterwachsen. Pilzbefund positiv.

27. II. Die Aussaat am Stamm ist reichlicher geworden und hat auch auf die Oberschenkel übergegriffen. Am Halse ein handbreiter Streifen, im Bereiche dessen die Haut diffus erkrankt ist. Auftreten von Bläschen an den Nagelwällen.

3. III. Nach Troekenlegung der Pat. Abheilung.

4. 41 jährige Frau mit Tbc. pulmon. Decubitis, Kachexie. Nach sechswöchentlichem Aufenthalt im Wasserbett erkrankt.

Status präsens: In der linken Kniegegend und der angrenzenden Partie des Oberschenkels einige bis linsengroße Bläschen. Pilzbefund positiv. Pat. kam ad exitum.

5. 42 jährige Frau mit Paraplegie der unteren Extremitäten. Guter Ernährungszustand. Erkrankte nach einmonatlichem Aufenthalt im Wasserbett.

Status praesens: Ergriffen sind in diffuser Weise die rechte Hohlhand und der zweite, dritte und vierte Interdigitalraum rechts. In der Gegend der linken Kniescheibe einige konfluierte Bläschen. Pilzbefund positiv. Abheilung durch Abschuppung ohne therapeutische Maßnahmen im Verlauf einer Woche nach Trockenlegung der Pat.

6. 47 jähriger Mann mit Paralysis progressiva. Schlechter Ernährungszustand. Nach neunwöchentlichem Aufenthalt im Wasserbett erkrankt.

Status praesens: Ergriffen in diffuser Weise beide Hohlhände und Beugeseiten der Endphalangen. Die Erkrankung ergreift auch beiderseits den zweiten, dritten und vierten Interdigitalraum. Pilzbefund positiv. Abheilung in einer Woche nach Trockenlegung des Pat.

7. 51 jährige Frau mit schweren Gelenksveränderungen infolge Rheumatismus. Sehr heruntergekommen. Seit drei Tagen im Wasserbett.

Status praesens: An den Beugeseiten der Finger links einige Bläschen.

Eine Woche später: Durch Aufschießen neuer Bläschen in der Umgebung der älteren sind über kronenstückgroße Herde entstanden. Auch die Vola manus und Interdigitalräume links sind ergriffen worden, einige Bläschen finden sich an der linken Thoraxseite. Pilzbefund positiv. Die Pat. kam ad exitum.

8. 73jährige Frau mit Decubitus. Guter Ernährungszustand. Erkrankt nach dreiwöchentlichem Aufenthalt im Wasserbett.

Status praesens: Bläschenform der Mykose. Ergriffen in mäßigem Grade beide Handteller und die meisten Interdigitalräume. Einzelne Bläschen auch an den Fußsohlen mit positivem Pilzbefund.

9. 36 jähriger Mann mit schweren arthritischen Gelenksveränderungen. Guter Ernährungszustand. Seit fünf Wochen im Wasserbett.

Status praesens: An beiden Handtellern und in den meisten Interdigitalräumen einzelstehende und $\mathrm{zu}$ Herden angeordnete, meist geplatzte Bläschen. Pilzbefund positiv.

10. 30jährige Frau mit Osteomyelitis. Guter Ernährungszustand. Seit 5 Wochen im Wasserbett.

Status praesens: In der Patellargegend und in der angrenzenden Partie des 
Oberschenkels beiderseits meist isoliert stehende bis linsengroße, flache, lebhaft rote Knötchen. Pilzbefund positiv.

11. 7jähriger Knabe mit Gastrostomiefistel. Guter Ernährungszustand.

Status praesens: An beiden Handtellern zahlreiche Bläschen und Blasenreste. In den Interdigitalräumen das Krankheitsbild einer interdigitalen Soormykose. Pilzbefund positiv.

Über den Verlauf der Wasserbettmykose sind nicht viel Worte zu verlieren. Unter gleichen äußeren Bedingungen bleibt die Erkrankung bei einigen Patienten auf eine kleine Körperstelle beschränkt, bei anderen ergreift sie in schubartigen Ausbrüchen einen großen Teil der Körperdecke. Lassen die Patienten über Nacht die Hände aus dem Wasser, so ist am Morgen bereits ein Abklingen der Erscheinungen unter Eintrocknung und stärkerer Abschuppung festzustellen. Eine Spontanheilung kommt auch bei Fortsetzung der Wasserbettbehandlung in einzelnen Fällen vor. Legt man die Patienten aufs Trockenbett, so heilt die Erkrankung ohne jede Behandlung in wenigen Tagen unter Abschuppung restlos ab. Dieser gutartige, zu Spontanheilung führende Verlauf ist insofern eigenartig, als die interdigitale Soormykose, die ja sowohl im klinischen Bilde als auch bezüglich des Erregers mit der in den Interdigitalräumen lokalisierten Wasserbettmykose identisch ist, meist recht hartnäckig ist.

Eine besondere geschlechtliche Disposition scheint nicht zu bestehen; daß bei Jacobi die Erkrankung auf der Männerstation häufiger auftrat und im Gegensatz dazu unter unseren Fällen sich mehr Frauen als Männer befanden, dürfte wohl nur in Zufälligkeiten gelegen sein.

Auch Alter und Ernährungszustand scheinen nach unseren Erfahrungen auf die Entstehung der Dermatose keinen Einfluß zu üben. Sie trat sowohl bei Patienten auf, die erst 3-4 Tage im Wasserbett lagen, als auch bei solchen, die schon monate- und jahrelang dieser Behandlung unterzogen wurden. Auch kam niemals eine Infektion des Wartepersonals vor.

Als Therapie kommt vor allem die Trockenlegung des Patienten in Betracht. Sollte dies undurchführbar sein, so wären Einpinselungen mit Teer zu versuchen.

Die Trichophytie Jacobis zeigt ein wesentlich anderes Krankheitsbild als unsere Fälle. Dort als Primärefflorescenzen ausschließlich dunkelrote, wenig infiltrierte Papeln, hier meist nur Bläschen, dort unter Größenwachstu m der einzelnen Efflorescenzen eine Ausbreitung der Erkrankung auf den ganzen Körper, hier ausschließlich Entstehung der Herde durch Apposition von Primärefflorescenzen, dort schließlich Ergiffenwerden aller dauernd im Wasser befind- 
lichen Hautpartien, hier sind gerade jene Stellen von der Mykose bevorzugt, die abwechselnd im warmen Wasser und an der Luft gehalten werden. Die von Jacobi und Küster beobachtete Dermatose kann zu höchst lästigen Komplikationen, wie schmerzhaften Rhagaden führen, in unseren Fällen ist das Leiden für den Patienten ziemlich harmlos, ja auch der Arzt muß öfters sehr darauf achten, daß er es nicht übersieht. Immerhin gibt es auch Übergänge zwischen beiden Erkrankungsformen wie Fall 3, der neben Bläschen an den Händen, am Stamme auch lebhaft rote Papeln als Primärefflorescenzen aufwies, die allerdings niemals ein Größenwachstum zeigten.

Und doch sind beide Erkrankungsformen, die von Jacobi und die an unserer Klinik beobachteten Fälle, was den Erreger betrifft, identisch. Die verschiedenen Bilder, die durch denselben Pilz in Freiburg und in Wien hervorgebracht werden, lassen sich entweder durch äußere Umstände oder die verschiedene Virulenz ein und desselben Pilzes erklären. Die erste Deutung verdient den Vorzug. Die Art der Wasserbettanlage, die Menge des Wasserinhaltes, der Wasserwechsel usw. scheint auf den Verlauf der Mykose nicht ohne Einfluß zu sein.

Die von Kraepelin abgebildeten und beschriebenen Dauerbäder bestehen eigentlich nur aus einer Badewanne mit verhältnismäßig geringem Rauminhalt, während an der Klinik Riehl das alte Hebrasche Modell mit kleinen Änderungen in Verwendung steht.

Es ist ein richtiges Bett, mit aufwindbarer Drahtmatraze, Kopf- und FuBstütze. Der Pat. liegt auf einer mit einem Leinentuch überzogenen Wolldecke, als Kopfunterlage dient ein Roßhaarpolster. Das Bett fa Bt beiläufig 8001 Wasser, das durch ständiges Nachlaufen täglich zweimal ersetzt wird, das Leintuch wird täglich, die übrige Wäsche alle 6 Tage gewechselt und gleichzeitig das Bett gründlich gereinigt. Schwerkranke Pat. entleeren Stuhl und Harn ins Wasser.

Es ist auch nicht auszuschließen, daß die wechselnde Virulenz des Pilzes in Freiburg und Wien die Verschiedenartigkeit des Krankheitsbildes hier und dort veranlaßt, stimmt doch diese Erklärung mit unseren Kenntnissen über die geographische Verbreitung der Pilze und die durch sie verursachten Erkrankungen sehr wohl überein. Andere Erklärungen für die Versehiedenartigkeit der von Jacobi und uns beobachteten Krankheitsbilder sind schon dadurch von der Hand zu weisen, daß in Freiburg die schwerere und in Wien nur die leichtere Form der Wasserbettmykose zur Beobachtung kam.

Über den Erreger der Wasserbettmykose können wir uns kurz fassen, da Küster bereits ausführlich darüber berichtet hat. In frisch erkrankten Fällen sind im Nativpräparate meist so massenhaft Pilze zu finden, wie kaum bei einer anderen Mykose. Man sieht so wie bei der interdigitalen Soormykose lange, dünne Fäden mit seitlichen und endständigen Aussprossungen und maulbeerartige Häufchen stark 
lichtbrechender, eiförmiger Gebilde. Besteht der Krankheitsherd bereits längere Zeit, so kann es vorkommen, daß man nur die maulbeerartigen Häufchen vorfindet, ja ab und zu waren in sicheren Krankheitsherden trotz mühevollen Suchens vorübergehend keine Pilzelemente nachweisbar. Wenn der Prozeß wieder aufflackert, dann ist der Pilzbefund auch wieder ein reichlicherer. Die Kültur, aus Schuppenpartikelchen auf Maltoseagar angelegt, gelingt leicht, doch wächst der Pilz in Fadenform erst, wenn man, ähnlich wie es $\mathrm{Küster} \mathrm{getan} \mathrm{hat,} \mathrm{ver-}$ schlechterte Wachstumsbedingungen schafft.

Der gefundene Pilz scheint mit der großsporigen Soorvarietät identisch zu sein, zumindest läßt er sich von dieser nicht abtrennen.

Jacobi hatte mit künstlichen Infektionsversuchen kein Glück. Auch wir brachten Reinkulturen des Pilzes in Wasserbetten, die mit mykosefreien Patienten belegt waren, ohne dadurch eine Erkrankung hervorzurufen. Es scheint eben eine besondere Disposition des Individuums zur Haftung des Pilzes notwendig zu sein, denn sonst wäre es nicht zu erklären, daß manche Patienten trotz jahrelangen Aufenthaltes im Wasserbett von dieser Erkrankung verschont bleiben. Die Pilze sind ja so verbreitet, daß sie auf der Haut eines jeden gesunden Individuums antreffbar sein dürften.

Der letzte Beweis, daß die Pilze tatsächlich die Ursache der Wasserbettmykose sind, ist nicht erbracht. Immerhin sind die Gründe, die man für die Erregernatur der Pilze anfübren kann - so insbesondere der Nachweis der Pilze in den Schuppen und das positive Kulturergebnis in allen dem klinischen Bilde nach hierhergehörigen Fällen, der Hinweis auf die Analogie mit der interdigitalen Soormykose usw. -, so schwerwiegend, daß an der Bedeutung der Pilze in ätiologischer Hinsicht kaum zu zweifeln ist.

Im histologischen Schnittpräparate konnten reichlich Pilzfäden in allen Lagen der Hornschicht nachgewiesen werden.

Es bleibt noch die Differentialdiagnose gegenüber anderen Erkrankungen zu besprechen. Sie ist im allgemeinen nicht schwer, um so mehr, als andere Hautkrankheiten im Wasserbette so gut wie nicht vorkommen, $\mathrm{ja}$ es ist gerade eine bisher noch nicht hinlänglich erklärte Eigenart dieser Behandlungsmethode, daß trotz der großen Infektionsmöglichkeit - die Patienten liegen doch im Wasser, in das sie ihre Exkremente und bei eiternden Prozessen oft literweise hochvirulenten Eiter entleeren -- eine Infektion oder ein Weiterschreiten eines bereits bestehenden eiternden Prozesses niemals vorkommt.

Nur ein ganz eigenartiges Ekzem kann man öfters beobachten, das bereits Hans $\nabla$. Hebra in einer Arbeit über Wasserbettbehandlung 1877 erwähnt. Da es leicht mit der Wasserbettmykose verwechselt werden kann, soll hier kurz darauf eingegangen werden. 
Das Ekzem tritt nach 8-14tägigem Aufenthalt im Wasserbett auf und lokalisiert sich an jenen Stellen der Haut, die, wenn der Patient im Wasserbett liegt, der Wasseroberfläche entsprechen, also am Nacken, in beiden Achselhöhlen und von da in einer Bogenlinie zum Nabel oder zur Sternalgegend. Außerdem wird auch oft die Haut in der Ellbogen- und Kniegegend davon befallen. Es treten winzig kleine, wenig erhabene, rote Papelchen auf mit einem entzündlichen Hof. Durch Aufschießen neuer Efflorescenzen and Weitergreifen der entzündlichen Rötung kommt es zur Konfluenz zu streifenförmigen oder unregelmäßig begrenzten Herden, die mäßiges Jucken verursachen. Die Begrenzung dieses Ekzems gegen die normale Haut ist unscharf. Die Ursache für das Auftreten dieses Ekzems ist wohl das Wasser, oder, genauer gesagt, der Reiz infolge Eintrocknen des Wassers. Daß die Beimengungen des Urins und Stuhls zum Badewasser ein unterstützendes Moment bilden, unterliegt keinem Zweifel, denn mit besonderer Vorliebe tritt es bei Patienten auf, die an Diarrhöe leiden. Der flüssige Stuhl mengt sich in kleinen Partikelchen mit dem Badewasser, steigt als spezifisch leichter an die Oberfläche, wird durch die Bewegungen des Patienten der Haut angelagert und trocknet dort ein, was sich öfters auch an einem gelben, abwaschbaren Streifen an der oben genannten Lokalisation erkennen läßt. Dieses Ekzem hat eine große Ähnlichkeit mit der papulösen Form der Wasserbettmykose, nicht nur was die Lokalisation betrifft. Sind Bläschen vorhanden, die beim Wasserbettekzem nicht vorkommen, oder lassen sich solche erschließen, wird die Differentialdiagnose leicht zu stellen sein. Sonst wäre noch zu verwerten, daß bei der Wasserbettmykose die einzelnen Papeln größer und vorspringender sind und daß sich leicht Schuppenmaterial zur Untersuchung gewinnen läßt, während beim Wasserbettekzem dies meist nicht gelingt. Schließlich bleibt als wichtigstes Differentialdiagnosticum der Pilznachweis.

Wasserbettmykose und Wasserbettekzem treten oft an einem Patienten gleichzeitig oder nacheinander auf, andererseits kommt auch jede der beiden Erkrankungen für sich allein zur Beobachtung.

Hängen so diese beiden Dermatosen auch vielfach zusammen, so wäre es doch verfehlt, das Wasserbettekzem bei andauernd negativem Pilzbefund mit der Mykose zu identifizieren.

\section{Iiteratur.}

1) Hebra, Allg. Wien: med. Zeitung 1861, Nr. 43. - 2) Hans v. Hebra, Wien. med. Wochenschr. 1877, Nr. 36, 37, 38, 39. - ${ }^{3}$ ) Krae peli n, Centralbl. f. Nervenheilk. u. Psych. 1901, S. 711. - ${ }^{4}$ ) Jacobi, Arch. f. Dermatol. u. Syphilis 84. 1907. - 5) Küster, Münch. med. Wochenschr. 1907, Nr. 32. - ${ }^{6}$ ) Küster Arch. f. Hyg. 62. 1907. - ${ }^{7}$ ) Ka uf mann Wolf, Dermatol. Zeitschr. 1915. 22. 8) Sacken, Mtt. a. d. Grenzgeb. d. Med. u. Chir. 32, H. 3. 1920. 